\title{
THE INFLUENCE OF SOCIAL FAMILY SUPPORT ON DEPRESSION, ANXIETY AND QUALITY OF LIFE AMONG WOMEN WITH BREAST CANCER : LITERATURE REVIEW
}

\author{
Tutik Rahayu ${ }^{1}$ Moses Glorino Rumambo Pandin ${ }^{2}$ \\ ${ }^{1}$ Student of Doctoral Program in Nursing, Faculty of Nursing, Airlangga University \\ ${ }^{2}$ Associate Professor, Faculty of Cultural Sciences, Airlangga University \\ Email: tutik.rahayu-2020@,fkp.unair.ac.id \\ Email : moses.glorino@,fib.unair.ac.id
}

\begin{abstract}
Background: Breast cancer is the second type of cancer diagnosed in women and is the first cancer in women worldwide. Breast cancer also causes high morbidity and mortality in women and becomes a heavy burden due to the incidence of disability due to the disease.

Purpose: This literature review aims to examine how social support affects anxiety, depression and quality of life in breast cancer sufferers.

Method: The data were obtained by searching for reputable and trustworthy journals. have high quality criteria, namely Scopus, Proquest, Science Direct, Elsevier, Pubmed. Journals or articles used in this review literature are searched using keywords and Boolean operators (AND, OR NOT, or AND NOT). Keywords in this review literature are as follows: social support OR family support, quality of life OR Quality, anxiety OR depression, AND Cancer OR cancer treatment OR Chronic disease.

Results: The results of this literature review show that there is a significant influence between the social support received by breast cancer patients on the improvement of their quality of life. The social support provided is also able to reduce anxiety and depression in breast cancer sufferers

Conclusion: Social support given to breast cancer patients is proven to have an effect on improving the quality of life, reducing anxiety and depression.

Keywords: social support, anxiety, depression, quality of life, breast cancer
\end{abstract}

\section{INTRODUCTION}

The number of cancer sufferers is currently increasing and the existence of new development program innovations for prevention such as early detection by screening and treatment has been able to increase the successful life expectancy of cancer sufferers, especially those who are still diagnosed with cancer at an early stage, however, most of them are survivors. continues to experience a decline in the quality of life related to Health even years after the completion of cancer treatment. Decreased quality of life includes chronic pain, fatigue, sexual dysfunction, and psychosocial problems such as depression, anxiety, and decreased social support. ${ }^{1}$

Breast cancer is the second type of cancer that is often diagnosed in women and is the number one cancer in women worldwide. Breast cancer also causes high morbidity and mortality rates in women and becomes a heavy burden due to the incidence of disability due to the disease. ${ }^{2}$ A conviction of being diagnosed with breast cancer not only affects the woman who is diagnosed but also has a big impact on those who are involved in the woman's life. ${ }^{3}$ Obtaining information related to this cancer diagnosis is a very sad thing in his life and feels that this is very likely to be close to death and suffering. ${ }^{4}$ Breast cancer patients can also experience both physical and 
psychological disorders or problems such as anxiety depression can be mild or severe this varies depending on the type and stage of cancer, prognosis, perception of post-treatment body image. ${ }^{4}$ relating to the illness and treatment they receive, so that it has an impact on aspects of life including work, finances, the future, activities and other social activities as well as the quality of their life ${ }^{2}$.

This cancer is also one of the causes of crisis for both the patient and the family, and is a major health problem which is generally fatal unless diagnosed early. ${ }^{5}$ Cancer diagnosis and early treatment can also reduce the quality of life for patients and their families. ${ }^{5}$

Quality of life is a multidimensional and complex concept that reflects patients' perceptions of the effect of diagnosis and initiating treatment on their daily lives. Quality of life is also related to how patients view subjectively from physical, psychological, social and spiritual aspects including well-being. High quality of life in breast cancer patients is associated with longer survival, besides that several factors such as self-readiness, financial, marriage, education level, age, hope for the future and social support are factors that affect the quality of life in breast cancer sufferers. ${ }^{6}$

One of the efforts that can be made to improve the quality of life in patients with breast cancer is social support. Social support is an experience received by someone related to the perception of support or voluntary support from others, from family, spouses, friends, relatives and social networks. ${ }^{7}$ Some forms of social support, namely informational support, are advice, suggestions, and information. Appraisal support is useful information for self-evaluation. Emotional support is classified as an expression of empathy, love, and care. Instrumental support is tangible assistance and services. ${ }^{7}$ Research results prove that there is a significant relationship between social support given to improving physical and psychological conditions in breast cancer patients reducing stress, depression, mortality and improving the quality of life of patients with cancer. ${ }^{8}$ Literature review This attempt to explain and analyze the effect of social support on improving the quality of life in breast cancer patients.

\section{METHOD}

The literature review relating to the social support provided to cancer patients to improve the quality of life is summarized thoroughly which will be presented in an article in the form of a Literature review. Completion of predetermined studies that are in accordance with the objectives of the literature review uses the PRISMA approach as a protocol and evaluation. Literature review is a summary based on several studies or studies that have been carried out previously with certain predetermined themes. The data used in this review literature is secondary data using sources of research results in previous studies. The data is obtained by searching for reputable and trustworthy journals. The search for journals is carried out from February to March 2021 using several databases that have high quality criteria, namely Scopus, Proquest, Science Direct, Elsevier, Pubmed,. The journals or articles used in this review literature are searched using keywords and Boolean operators (AND, OR NOT, or AND NOT) this is done to expand the detail or specific search for articles according to the purpose and can make it easier to determine the articles or journals used. Keywords in this review literature are as follows: social support OR family support, quality of life OR Quality, anxiety OR depression, AND Cancer OR cancer treatment OR Chronic 
The inclusion criteria are full text, from 2019 to 2021, after going through the screening process, there were 57 articles and journals and journal searches using the PICOS framework strategy, namely: found 13 journals that match the criteria consisting of Population / Problem, namely cancer patients undergoing care in a hospital or health service center, Intervention is a patient who gets family or social support, Comparation is no comparison., Outcome is the quality of life in cancer patients who get social or family support, Study design is Quasi experimental studies, randomized control and trial, crossectional studies

\section{RESULT AND DISCUSION}

\section{Table 1}

\section{Article Summary About The Effect Of Social Family Support On Depression, Anxiety And Quality Of Life Among Women With Breast Cancer: Literature Review}

\begin{tabular}{|c|c|c|c|}
\hline Authors, years, title & $\begin{array}{l}\text { Study design, Sample, } \\
\text { Variable, Instrument, } \\
\text { Analysis }\end{array}$ & $\begin{array}{l}\text { Outcome of Analysis } \\
\text { Factors }\end{array}$ & Summary of Result \\
\hline $\begin{array}{l}\text { Authors : Aomei Shena, } \\
\text { Wanmin Qianga,*, } \\
\text { Ying Wanga, Yuhong } \\
\text { Chenb } \\
\text { Years : } 2020 \\
\text { Title : Quality of life } \\
\text { among breast cancer } \\
\text { survivors with triple } \\
\text { negative breast } \\
\text { cancer-role of hope, } \\
\text { self-efficacy and social } \\
\text { support } \\
2\end{array}$ & $\begin{array}{l}\text { Study design : cross- } \\
\text { sectional study } \\
\text { Sample : } 121 \\
\text { participants } \\
\text { Variable : Quality of } \\
\text { life, } \\
\text { hope, self-efficacy, } \\
\text { social support } \\
\text { Instrument : Herth } \\
\text { Hope Index (HHI), } \\
\text { Social Support Rating } \\
\text { Scale (SSRS), General } \\
\text { Self-Efficacy Scale } \\
\text { (GSES), Functional } \\
\text { Assessment of Cancer } \\
\text { Therapy Breast Cancer } \\
\text { (FACT-B) } \\
\text { Analysis : ANOVA } \\
\text { analysis }\end{array}$ & $\begin{array}{l}\text { Hope, social support } \\
\text { and self-efficacy were } \\
\text { all positively } \\
\text { correlated with } \\
\text { quality of life ( } \mathrm{P}< \\
0.001) \text {. Multiple } \\
\text { regression analyses } \\
\text { indicated hope, } \\
\text { income, cancer stage, } \\
\text { self-efficacy, and } \\
\text { social support as } \\
\text { indicators of quality } \\
\text { of life, explaining } \\
56.2 \% \text { of the response } \\
\text { variation (P }<0.001) \text {. }\end{array}$ & $\begin{array}{l}\text { Quality of life of triple negative } \\
\text { breast cancer patients need to be } \\
\text { improved. Income, hope, self- } \\
\text { efficacy and social support are } \\
\text { positive predictors, and cancer } \\
\text { stage are negative predictors of } \\
\text { quality of life. Supportive } \\
\text { programs and interventions } \\
\text { targeting on increasing levels of } \\
\text { hope, self efficacy and social } \\
\text { support should be considered } \\
\text { while caring for this group. }\end{array}$ \\
\hline $\begin{array}{l}\text { Authors: } \\
\text { Hadi Zamanian, } \\
\text { Mohammadali Amini- } \\
\text { Tehrani, Zahra Jalali, } \\
\text { Mona Daryaafzoon, } \\
\text { Sara Ala, Samira } \\
\text { Tabrizian, Sahar } \\
\text { Foroozanfar } \\
\text { Years: } 2020 \\
\text { Title : Perceived social } \\
\text { support, coping } \\
\text { strategies, anxiety and } \\
\text { depression among } \\
\text { women with breast } \\
\text { cancer: Evaluation of a } \\
\text { mediation model } \\
3\end{array}$ & $\begin{array}{l}\text { Study design : cross- } \\
\text { sectional study } \\
\text { Sample : } 221 \\
\text { participant } \\
\text { Variable: Perceived } \\
\text { social support, Coping } \\
\text { strategies, } \\
\text { Anxiety, depression, } \\
\text { Instrument : } \\
\text { Depression-Anxiety- } \\
\text { Stress (DASS-21) scale, } \\
\text { social support was } \\
\text { measured using the } \\
\text { Medical Outcome } \\
\text { Survey, Social Support } \\
\text { Scale (MOSS-SSS), } \\
\text { and coping strategies }\end{array}$ & $\begin{array}{l}\text { All of the social } \\
\text { support subscales } \\
\text { were negatively } \\
\text { correlated with } \\
\text { depression and } \\
\text { anxiety. Most of the } \\
\text { coping strategies } \\
\text { were negatively } \\
\text { correlated with } \\
\text { depressive-anxious } \\
\text { symptoms. When } \\
\text { controlling for } \\
\text { covariates, mediation } \\
\text { analysis revealed that } \\
\text { active coping, } \\
\text { positive reframing } \\
\text { and acceptance } \\
\text { partially mediated the }\end{array}$ & $\begin{array}{l}\text { High levels of social support may } \\
\text { relieve depressive and anxious } \\
\text { symptoms of breast cancer } \\
\text { patients through functional coping. } \\
\text { The management of breast cancer } \\
\text { patients should also focus on } \\
\text { providing patients with social } \\
\text { support and educating them on the } \\
\text { practice of functional coping } \\
\text { strategies.. }\end{array}$ \\
\hline
\end{tabular}




Authors: Gabriela
Toledo \& Carol Y.
Ochoa \& Albert J.
Farias
Years: 2019
Title: Exploring the role
of social support and
adjuvant endocrine
therapy
use among breast
cancer survivors

Authors : Awolu Adam, Felix Koranteng

Years: 2020

Title: Availability, accessibility, and impact of social support on breast cancer treatment among breast cancer patients in Kumasi, Ghana: A qualitative study were measured using the brief COPE Analysis: Backward stepwise regression analysis association of social support of different types with depression. Positive reframing also partially mediated the relationship of the total social support and positive social interactions with anxiety.

Study design: Kualitatif Sample: 22 partisipan Instrument: conducted semi-structured indepth interviews Analysis: analyzed interview transcripts with a grounded theory approach and grouped social support into four categories (emotional, informational, instrumental, and appraisal), then used a thematic content analysis to identify the sources and delivery of support

Study design:

Qualitative Study Sample: 15 partisipan Variable: Availability, accessibility, social support

Instrument: Dengan pertanyaan semi terstruktur melalui wawancara mendalam Analysis: Thematic analysis

\section{Women described} that informational support was provided by medical providers who explained the purpose, benefits, and management of AET. Emotional support in the form of reassurance, communication, and empathy was provided by family, survivorship groups, medical providers, and spirituality/religiosity. Women identified several organizations and exercises that provided them with instrumental and appraisal support in the form of physical and emotional benefits, which was also provided by family, friends, and medical providers.

Majority of the women who participated in the study were postmenopausal women with an average age of 55 years. The study also revealed that all the participants in this study received one kind of support or another including informational,
We identified that social support delivered to breast cancer survivors provided women with educational, physical, and emotional benefits that may play an important role in their continuation of AETa.
Social support is critical for the survival and quality of life of chronic disease patients including breast cancer patients who were the focus of this study. The availability and/or accessibility of social support or otherwise significantly determines the prognosis and quality of life of breast cancer patients. Healthcare professionals and family members or significant others are major players in organizing social support for chronic disease patients. 
Authors : Aylin Aydin Sayılan, Melike Demir Doğan

Years: 2019

Title: Illness perception, perceived social support and quality of life in patients with diagnosis of cancer
Authors : Rahel

Aberaraw, Abdisa

Boka, Roza Teshome, Addisu Yeshambel

Years: 2020

Title: Social networks and quality of life among female breast cancer patients at Tikur Anbessa specialized hospital, Addis Ababa, Ethiopia 2019 financial, emotional, and tangible support and reported varying positive impacts on their lives as a result of the support received. For those who received support, the prognosis and general quality of life appeared promising and well-adjusted than those who reported not having received any form of support.

Study design: crosssectional study Sample: 186 partisipan Variable: Illness, social support, quality of life Instrument:

Multidimensional

Perceived Social

Support Scale, the Illness

Perception

Questionnaire (IPQ) and the Nightingale Symptom Assessment Scale (NSAS)

Analysis: t test,

Pearson's rho

correlations

Study design: crosssectional study Sample: 214 partisipan Variable: Social networks, quality of life Instrument: EORTC, QLQ

Analysis: multivariate Analysis, logistic regression
The mean age of the patients in the study was $61.37 \pm 14.89$ years. Married patients had better social support than single individuals $(\mathrm{t}=$ 2.129; $\mathrm{p}=0.035)$.

Significant negative correlation was found between the illness perception scale and total quality of life scale score $(\mathrm{r}=$ $-0.163 ; p=0.026)$, and physical wellbeing $(r=-0.224 ; p=$ $0.002)$ and social well-being $(\mathrm{r}=$ $-0.199 ; \mathrm{p}=0.006$ ) subscale

A total of 214 females with breast cancer were recruited with a mean age of 41.85. Participants who had children $(\mathrm{AOR}=5,95 \% \mathrm{CL}$ : 1.3,21 COR =6), and other relatives (AOR $=6,95 \% \mathrm{CI}: 1.2,30$, COR $=7$ ), were more likely to have good social networks. Participants who were not married $(\mathrm{AOR}=$ $0.02,95 \% \mathrm{CI}: 0.03$, $0.28)$, had no parents living $(\mathrm{AOR}=0.1$, 95\%CI: $0.02,0.4)$, no
Patients' social support and quality of life were at moderate levels. Married participants had better social support than single participants, and operated participants had better social support than unoperated participants. As illness perception decreased, quality of life, physical well-being and social well-be he quality of life was relatively low and social support were found to be poor in women with breast cancer. Health-care providers in oncology departments need to focus on addressing the side effects of therapy and social networks which may help to improve the quality of life of females with breast cancer 


Authors : Gülden
Küçükakça Çelik,
Hatice Çakır, Engin Kut
Years: 2020
Title: Mediating Role of
Social Support in
Resilience and Quality
of Life in Patients
with Breast Cancer:
Structural Equation
Model Analysis

Authors : Amelie G.

Ramirez, DrPH 1;

Byeong Yeob Choi;

Edgar Munoz; Arely

Perez; Kipling J.

Gallion; Patricia I.

Moreno; and Frank J.

Penedo

Years: 2019

Title: Assessing the

Effect of Patient

Navigator Assistance

for

Psychosocial Support

Services on Health-

Related Quality of

Life in a Randomized

Clinical Trial in Latino

Breast, Prostate,

and Colorectal Cancer

Survivors close friends $(\mathrm{AOR}=$ 0.06, 95\%CI: 0.01, $0.4)$, and no neighbors $(\mathrm{AOR}=$ 0.09 , 95\%CI: 0.03, $0.5)$ had poor social networks.

Study design: cross sectional study

Sample: 113 sampel

Variable: MPSSS,

(EORTC QLQ-C30), EORTC QLQ-BR23, GHS, QoL scale, symptom scales (SCs) Analysis: Statistical Package for Social Sciences (SPSS) for Windows 23.0 (SPSS Inc, Chicago, Illinois) and Analysis of Moment Structures 21.0

Study design:

Randomized Clinical Trial

Sample: 288 sample

Variable: Patient

Navigator, Health-

Related Quality of

Life

Instrument: Charlson

Comorbidity Index, Functional Assessment of Cancer TherapyGeneral (FACT-G) scale Analysis: SAS PROC PLAN software (version 9.2
: Social support played a partial mediator role in the relationship between resilience and functional QoL.

There was a negative correlation between functional QoL Questionnaire Breast Cancer 23 and psychological resilience and social support $(\mathrm{P}<0.005)$. The mediation effect ratio was $10.2 \%$ (R2 $=0.102$ ). Social support was found to not have a mediating role in the relationship between psychological resilience and general QoL $(\mathrm{P}<0.05)$

\section{PN-LCNS}

demonstrated significant improvement HRQOL comparison with $\mathrm{PN}$ only for colorectal cancer survivors but not for breast and prostate cancer survivors.
Patients do not want social support to end, and their weakness in the eyes of others may have a negative impact on their QoL and resilience
Enhanced PN improves HRQOL among Latino colorectal cancer survivors. Future research should identify the best strategies for engaging Latino survivors in $\mathrm{PN}$ programs. PN programs should also be adapted to address HRQOL concerns among Latina breast cancer survivors. Cancer 2020;126:1112-1123. (C) 2019 The Authors. Cancer published by Wiley Periodicals, Inc. on behalf of American Cancer Society. This is an open access article under the terms of the Creative Commons Attribution-NonCommercial-

NoDerivs License, which permits use and distribution in any medium, provided the original work is properly cited, the use is non-commercial and no modifications or adaptations are made. 


Authors : Mojgan
Firouzbakhta,
Karimollah Hajian-
Tilakib, Dariush
Moslemic
Years: 2020
Title: Analysis of
quality of life in breast
cancer survivors using
structural
equation modelling: the
role of spirituality,
social support and
psychological well-
being

Authors : Nuworza

Kugbey, Kwaku

Oppong Asante, Anna

Meyer-Weitz

Years: 2019

Title: Depression, anxiety and quality of life among women living

with breast cancer in Ghana: mediating roles of social support and religiosity
Study design: crosssectional study Sample: 305 sample Variable: quality of life, spirituality, social support and psychological Instrument: The standard European Organization for Research and Treatment of Cancer (EORTC) QLQ-C30 QoL scale, a system-of-belief inventory questionnaire, the social support scale, the short form of the Hospital Anxiety and Depression Scale and the fatigue severity scale (FSS)

Analysis: SPSS version 18.0

(SPSS, Chicago, IL, USA) and SPSS Amos 24 (IBM, Armonk, NY, USA)

Study design: crosssectional study Sample: 205 sample Variable: Depression, anxiety, quality of life, social support, religiosity Instrument: Functional assessment of cancer therapy - breast cancer (FACT-B), Multidimensional Perceived Social Support Scale, Santa Clara Strength of Religious Faith Questionnaire, Hospital Anxiety and Depression Scale

Analysis: (SPSS) with PROCESS

Macro
The median age (quartile 1 [Q1], quartile 3 [Q3]) of patients was 50 y (43, 55). The psychological factors designated by anxiety, depression and FSS had a negative significant direct effect on QoL $(\beta=-0.62)$.

Spirituality has a positive direct effect ( $\beta=0.089$ ) but a negligible indirect effect $(\beta=0.020)$ on QoL, while the direct association of social support was almost negligible..

Findings showed that there were significant direct negative effects of depression and anxiety on quality of life. Whereas depression had a significant negative indirect effect on quality of life through social support $(b=-0.247,95 \%$ $\mathrm{CI}=-0.482$ to $-0.071)$, anxiety had a significant positive indirect effect on quality of life through social support $(\mathrm{b}=0.142$, $95 \% \mathrm{CI}=0.011$ to 0.324). However, depression and anxiety did not have any significant indirect effect on quality of life through religiosity
The findings emphasized the unifying structure of the determinants of QoL and the mediating negative association of psychological factors with QoL. Thus the supportive education efforts should focus on improving psychological well-being along with standard treatment in breast cancer survivors.

These findings suggest that there is the need to examine the mental health of women living with breast cancer as part of the routine healthcare, and social support ties should be strengthened to improve their quality of life. 
Authors : Aomei Shen, Wanmin Qiang, Ying Wang, Yuhong Chen

Years: 2020

Title: Quality of life among breast cancer survivors with triple negative breast cancer-role of hope, self-efficacy and social support
Authors : Jie Zhao, Yong Ma, Tetsuya Tanimoto, Akihiko Ozaki, Wan-Li Chen, Jing-Ya Wang, Yu-Xin Zhang, Lin-Li Chen, Ji-Wei Wang, Jin-Ming Yu1

Years: 2020

Title: Effects of physical activity and stress on the relationship between social capital and quality of life among breast cancer survivors
Study design: crosssectional study Sample: 121 sample Variable: Quality of life hope, self-efficacy, social support Instrument: Herth Hope Index (HHI), Social Support Rating Scale (SSRS), General SelfEfficacy Scale (GSES), Functional Assessment of Cancer Therapy Breast Cancer (FACTB) Analysis: ANOVA

Study design: crosssectional study Sample: 532 sample Variable: social capital, physical activity, perceived stress, QOL. Instrument: cognitive and structural domains by the scale, the health-promoting lifestyle profile-II, the Perceived Stress Scale (PSS-14), European Organization for Research and Treatment of Cancer (EORTC) Analysis: T-test, ANOVA
Hope, social support and self-efficacy were all positively correlated with quality of life $(\mathrm{P}<$ 0.001). Multiple regression analyses indicated hope, income, cancer stage, self-efficacy, and social support as indicators of quality of life, explaining $56.2 \%$ of the response variation $(\mathrm{P}<0.001)$.

The separate
mediations of two
single mediating
variables were found
signifcant in the
relationship of QOL
with control over life
and feeling about the
community. In the
relationship of QOL
with social
participation, social
network and social
support, the separate
mediation of physical
activity was
signifcant, while the
separate mediation of
perceived stress was
not signifcant. AS.

: Quality of life of triple negative breast cancer patients need to be improved. Income, hope, selfefficacy and social support are positive predictors, and cancer stage are negative predictors of quality of life. Supportive programs and interventions targeting on increasing levels of hope, self efficacy and social support should be considered while caring for this group

A multidisciplinary team approach and a variety of delivery systems are needed to address the social, physical and psychological issues for improving QOL among BCSs.

The results of this literature review show that social support provided to patients with breast cancer can improve their quality of life and reduce anxiety and depression. Physical activity and perceived stress also have a significant effect on quality of life. ${ }^{9}$ Results Previous studies found that the problems identified in many cancer patients were depression, anxiety and pain or discomfort. The existence of this condition should be handled not only aimed at overcoming physical problems but also the impact of the sick conditions suffered by cancer patients such as anxiety, depression and discomfort should be a concern and an important factor for getting good care that involves collaborative health workers. ${ }^{10}$ Social stigma was also associated with worsening quality of life in cancer patients. ${ }^{11}$ The findings in this study also indicate that age has a direct positive effect on comorbidity, and that comorbidity as a biologically mediating variable has a significant negative effect on quality of life. ${ }^{12}$ Patients with advanced cancer also show a decrease in quality of life due to the impact of cancer, such as decreased physical function. ${ }^{13}$ Providing social support after treatment for patients with cancer also affects their quality of life. 
The study results also showed a positive relationship between social support received and the improvement of the patient's quality of life, such as physical and psychological aspects. Support has an important mediating role in improving the readiness and quality of life of breast cancer patients, patients who report that the support they receive can be obtained forever and does not end. ${ }^{14}$ The research in this literature also explains the findings of low social support for decreased quality of life. ${ }^{15}$ provided among them, namely emotional support, informational, and instrumental and real support. Their social support comes from various sources, namely family, hospitals, friends, places of worship and various other sources. ${ }^{16}$ The results shown in an important study of the social support received by patients with breast cancer are nurses. The nurse provides important information regarding the condition of the disease and provides motivation and encouragement and this makes the patient feel calmer. Informational support is also obtained from health workers, namely doctors related to the therapy to be undertaken, nutrition that must be consumed, medication and self-care that can be done. They also received information about the effect or impact that would be received from cancer therapy or treatment, such as hair loss, weight loss and appetite. ${ }^{16}$

The findings in this study are in line with the results of a qualitative study that provided the informational support found in women with breast cancer receiving endocrine therapy. This informational support is provided by health workers by providing information related to the duration and duration of treatment, the effects of treatment and the benefits that will be received from the treatment being carried out. ${ }^{7}$ Emotional support provided by health workers to women with breast cancer can also provide a positive outlook. on current therapies and their future. Instrumental support is also received from the family of friends and friends by offering responsible assistance and having a commitment to providing support, including financial. ${ }^{7}$ Social support is perceived as a feeling of worth being loved and respected. Social support can reduce the adverse effects of quality of life and depression. ${ }^{17}$

The results of research in this literature review also describe patients with breast cancer who experience problems with anxiety and depression, as well as the physical and psychological burdens they receive from the treatment they are given and the presence of a sense of concern for their future health and the fate of their close family. ${ }^{18}$ This has an impact on the deteriorating quality of life. Women with breast cancer tend to have mental health problems with a high prevalence such as depression and anxiety. ${ }^{10}$ Factors of anxiety and depression experienced by women with breast cancer are one of the factors that significantly reduce the overall quality of life. ${ }^{10}$ Breast cancer is one of the factors The causes of changes in sufferers include daily activities, social relationships, work, family roles and psychological stress. This situation has an impact on the lack of personal and social control, decreased physical health and death. This situation can lead to depression and social isolation so that support from family, friends, social networks and health service providers is needed by breast cancer sufferers. Empiric evidence shows that social support provided by family, relatives, friends is able to improve behaviors such as diet, physical activity and adherence to treatment. ${ }^{19}$ Social support can help improve self-protection in dealing with stressful conditions, stress, depression due to cancer. them. ${ }^{20}$ The research results also prove that the support provided by nurses is able to improve resilience in women who suffer from cancer. ${ }^{21}$ Cancer patients with an age of more than 50 years are proven to have an increase in the quality of 
life with the social support they receive. ${ }^{22}$ Breast cancer sufferers who experience metastases reveal the meaning of life and hope for the acceptance of social support and spirituality. ${ }^{23}$

Quality of life is related to complex health problems which are viewed from various multidimensional aspects, both physical, psychological and social. ${ }^{24}$ Subjective quality of life has been recognized as an indicator of health status for cancer sufferers. The lack of social support is one of the factors that worsens it. The quality of life for people with cancer. ${ }^{25}$ Is breast cancer and this is influenced by several factors such as values, norms, culture, ethnicity and their experiences. Some of the successes of cancer treatment have been shown to improve the survival of cancer patients, but it still raises concerns about the side effects of treatment such as infertility, menopausal symptoms, recurrence and recurrent malignancy. Health perceptions in terms of quality of life are also influenced by several factors such as social demographics. , biological factors, spiritual, social support, psychological conditions and lifestyle. ${ }^{12}$

\section{CONCLUSION}

Breast cancer is still the most common cause of death in women. Various effects of the disease are felt by breast cancer sufferers both physically, such as pain and discomfort, psychological in the form of anxiety and depression, and social, for example in work, relationships with family, relatives and friends. These various impacts have been shown to significantly affect the quality of life for breast cancer sufferers. One of the efforts that can be made to improve the quality of life for breast cancer sufferers is to provide social support. The social support received by the sufferer is felt as a form of appreciation, affection is valued and respected. The social support provided can be in the form of emotional support, informational support and instrumental support. Nurses have an important role in improving the quality of life, reducing anxiety and depression in cancer patients by providing support to breast cancer sufferers.

\section{REFERENCES}

1. Ramirez AG, Choi BY, Munoz E, et al. Assessing the effect of patient navigator assistance for psychosocial support services on health-related quality of life in a randomized clinical trial in Latino breast, prostate, and colorectal cancer survivors. Cancer. 2020;126(5):1112-1123. doi:10.1002/cncr.32626

2. Fernández de Larrea-Baz N, Pérez-Gómez B, Guerrero-Zotano Á, et al. Primary breast cancer and health related quality of life in Spanish women: The EpiGEICAM case-control study. Sci Rep. 2020;10(1):1-13. doi:10.1038/s41598-020-63637-w

3. Aberaraw R, Boka A, Teshome R, Yeshambel A. Social networks and quality of life among female breast cancer patients at Tikur Anbessa specialized hospital, Addis Ababa, Ethiopia 2019. BMC Womens Health. 2020;20(1):1-9. doi:10.1186/s12905-020-00908-8

4. Zamanian H, Amini-Tehrani M, Jalali Z, et al. Perceived social support, coping strategies, anxiety and depression among women with breast cancer: Evaluation of a mediation model. Eur J Oncol Nurs. 2021;50(November 2020):101892. doi:10.1016/j.ejon.2020.101892

5. Aydın Sayılan A, Demir Doğan M. Illness perception, perceived social support and 
quality of life in patients with diagnosis of cancer. Eur J Cancer Care (Engl). 2020;29(4):1-7. doi:10.1111/ecc.13252

6. Shen A, Qiang W, Wang Y, Chen Y. Quality of life among breast cancer survivors with triple negative breast cancer--role of hope, self-efficacy and social support. Eur J Oncol Nurs. 2020;46(December 2019):101771. doi:10.1016/j.ejon.2020.101771

7. Toledo G, Ochoa CY, Farias AJ. Exploring the role of social support and adjuvant endocrine therapy use among breast cancer survivors. Support Care Cancer. 2020;28(1):271-278. doi:10.1007/s00520-019-04814-0

8. Osann K, Wilford J, Wenzel L, et al. Relationship between social support, quality of life, and Th2 cytokines in a biobehavioral cancer survivorship trial. Support Care Cancer. 2019;27(9):3301-3310. doi:10.1007/s00520-018-4617-z

9. Zhao J, Ma Y, Tanimoto T, et al. Effects of physical activity and stress on the relationship between social capital and quality of life among breast cancer survivors. Sci Rep. 2020;10(1):1-12. doi:10.1038/s41598-020-74706-5

10. Khue PM, Thom VT, Minh DQ, Quang LM, Hoa NL. Depression and anxiety as key factors associated with quality of life among lung cancer patients in Hai Phong, Vietnam. Front Psychiatry. 2019;10(MAY):1-7. doi:10.3389/fpsyt.2019.00352

11. Zhang Y, Cui C, Wang Y, Wang L. Effects of stigma, hope and social support on quality of life among Chinese patients diagnosed with oral cancer: A cross-sectional study. Health Qual Life Outcomes. 2020;18(1):1-8. doi:10.1186/s12955-020-01353-9

12. Firouzbakht M, Hajian-Tilaki K, Moslemi D. Analysis of quality of life in breast cancer survivors using structural equation modelling: The role of spirituality, social support and psychological well-being. Int Health. 2020;12(4):354-363.

doi:10.1093/INTHEALTH/IHZ108

13. Johnson LA, Schreier AM, Swanson M, Moye JP, Ridner S. Stigma and quality of life in patients with advanced lung cancer. Oncol Nurs Forum. 2019;46(3):318-328. doi:10.1188/19.ONF.318-328

14. Celik G, Çakır H, Kut E. Mediating Role of Social Support in Resilience and Quality of Life in Patients with Breast Cancer: Structural Equation Model Analysis. Asia-Pacific J Oncol Nurs. 2021;8(1):86. doi:10.4103/apjon.apjon_44_20

15. Geue K, Götze H, Friedrich M, et al. Perceived social support and associations with health-related quality of life in young versus older adult patients with haematological malignancies. Health Qual Life Outcomes. 2019;17(1):1-10. doi:10.1186/s12955-0191202-1

16. Adam A, Koranteng F. Availability, accessibility, and impact of social support on breast cancer treatment among breast cancer patients in Kumasi, Ghana: A qualitative study. PLoS One. 2020;15(4):1-16. doi:10.1371/journal.pone.0231691

17. Kong LN, Hu P, Yao Y, Zhao QH. Social support as a mediator between depression and quality of life in Chinese community-dwelling older adults with chronic disease. Geriatr Nurs (Minneap). 2019;40(3):252-256. doi:10.1016/j.gerinurse.2018.10.014 
18. Sodergren SC, Wheelwright SJ, Permyakova N V., et al. Supportive care needs of patients following treatment for colorectal cancer: risk factors for unmet needs and the association between unmet needs and health-related quality of life-results from the ColoREctal Wellbeing (CREW) study. J Cancer Surviv. 2019;13(6):899-909. doi:10.1007/s11764019-00805-6

19. Srivastava J, Kaushik S, Tewari M, Shukla H. Mediating role of affective experiences in the relationship between perceived social support and life satisfaction among breast cancer patients. Indian J Palliat Care. 2021;27(1):76-82. doi:10.4103/IJPC.IJPC_106_20

20. Vodermaier A, Linden W. Social support buffers against anxiety and depressive symptoms in patients with cancer only if support is wanted: a large sample replication. Support Care Cancer. Published online 2019:2345-2347. doi:10.1007/s00520-019-04737$\mathrm{W}$

21. Kofler S, Kobleder A, Ott S, Senn B. The effect of written information and counselling by an advanced practice nurse on resilience in women with vulvar neoplasia six months after surgical treatment and the influence of social support, recurrence, and age: A secondary analysis of a multicenter randomized controlled trial, WOMAN-PRO II. BMC Womens Health. 2020;20(1):1-9. doi:10.1186/s12905-020-00965-z

22. Kobayashi R, Ishizaki M. Relationship between health literacy and social support and the quality of life in patients with cancer: Questionnaire study. J Particip Med. 2020;12(1):112. doi:10.2196/17163

23. Adler SR, Coulter YZ, Stone K, Glaser J, Duerr M, Enochty S. End-of-Life Concerns and Experiences of Living With Advanced Breast Cancer Among Medically Underserved Women. J Pain Symptom Manage. 2019;58(6):959-967. doi:10.1016/j.jpainsymman.2019.08.006

24. Wang G, Yang Q. Factors associated with health-related quality of life in Chinese patients with heart failure: a cross-sectional study. Collegian. 2020;27(5):512-520. doi:10.1016/j.colegn.2020.04.003

25. Mamguem Kamga A, Dumas A, Joly F, et al. Long-Term Gynecological Cancer Survivors in Côte d'Or: Health-Related Quality of Life and Living Conditions. Oncologist. 2019;24(7):490-500. doi:10.1634/theoncologist.2018-0347 